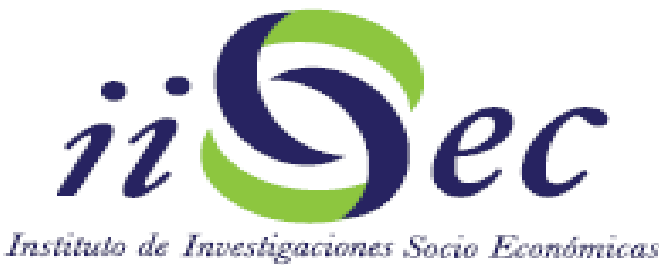

Documento de Trabajo No. 02/15

Febrero 2015

Disponibilidad, Consumo y Utilización

Biológica de Alimentos en Bolivia:

Análisis y Perspectivas (1990-2030)

por:

Blanca Rivero Lobo

Javier Aliaga Lordemann 


\title{
Disponibilidad, consumo y utilización biológica de alimentos en Bolivia: Análisis y Perspectivas (1990-2030)
}

\author{
Blanca Rivero Lobo* \\ Javier Aliaga Lordermann**
}

\section{Resumen:}

El presente documento, evalúa cuatro dimensiones de la Seguridad Alimentaria (i.e. disponibilidad, soberanía, estabilidad y consumo y utilización biológica).Encontrando brechas para los grupos de alimentos de: frutas y verduras, proteínas y carbohidratos, a partir de comparar la oferta de cada producto con los requerimientos nutricionales mínimos de la población boliviana en el periodo de 1990 al 2012.

El resultado más relevante de la investigación es que por grupo de alimento, sólo el grupo de carbohidratos cumple con las dimensiones estudiadas. Tanto el grupo de proteínas, como el de frutas y verduras, no cumplen con ninguna dimensión.

También se evidencia que existen factores culturales que inciden sustancialmente en la alimentación. Así mismo no existen incentivos, ni políticas de seguridad alimentaria a largo plazo, por lo que se genera una situación de vulnerabilidad. Estas brechas nutricionales son indicadores de desnutrición y desabastecimiento de alimentos.

Palabras Clave: Seguridad Alimentaria Nutricional, Inseguridad Alimentaria, Seguridad Alimentaria y Soberanía Alimentaria.

Código JEL.: Q11, Q18, Y40.

\section{Abstract:}

This paper evaluates four dimensions of food security (ie availability, sovereignty, stability and consumption and biological utilization). Finding gaps for food groups: fruits and vegetables, proteins and carbohydrates, from comparing the supply of each product with minimum nutritional requirements of the Bolivian population in the period 1990 to 2012 .

The most important result of the research is that food group, only the carbohydrate group meets the dimensions studied. Both groups of proteins, such as fruits and vegetables do not meet any dimension.

Is also evidence that there are cultural factors that materially affect the feed. Likewise, there are no incentives or policies on food security in the long term, so that a position of vulnerability is generated. These nutritional gaps are indicators of malnutrition and food shortages.

Key Words: Food Security and Nutrition, Food Insecurity, Food Security and Food Sovereignty.

JEL Classification Numbers: Q11, Q18, Y40.

\footnotetext{
Asistente de Investigación del Instituto de Investigaciones Socio Económicas de la Universidad Católica Boliviana "San Pablo". Contacto: blanka16@hotmail.com

** Director del Instituto de Investigaciones Socio Económicas de la Universidad Católica Boliviana "San Pablo". Contacto: jaliaga@ucb.edu.bo
} 


\section{Disponibilidad, consumo y utilización biológica de alimentos en Bolivia: Análisis y Perspectivas (1990-2030)}

\section{$1 \quad$ Introducción}

Las estimaciones más recientes de la FAO (2013) indican que la gran mayoría (827 millones de personas que padecen hambre) vive en regiones en desarrollo, donde la prevalencia de la subalimentación en el periodo 2011-2013 se estima actualmente en el $14,3 \%$ de la población. Para Mallea (2010) Bolivia está clasificada como un país con alta vulnerabilidad a la inseguridad alimentaria (IA) y con alto grado de pobreza.

Haquim, D. (2009) explica que existe poco acceso de los hogares a los alimentos en un país en el cual el $47 \%$ de la población es pobre y el $76 \%$ de los pobres quienes viven en el área rural no tiene acceso a los servicios básicos ni de infraestructura. En el ranking sobre vulnerabilidad a la IA, elaborado por UDAPE (2007) se demuestra que las comunidades son el sector más vulnerable del país representando alrededor del $53 \%{ }^{1}$.

Según el perfil nutricional realizado por la FAO/WHO/UNU (2001) para Bolivia, se demuestra que las ciudades más densamente pobladas presentan condiciones de desnutrición. Dentro del estado nutricional de los niños menores de 5 años resalta un retardo de crecimiento e insuficiencia ponderal. Importantes diferencias en los niveles de desnutrición en menores de 5 años persisten entre las regiones. La región del Llano es la que presenta los mejores indicadores, mientras que en el Altiplano se encuentran los niveles más altos de desnutrición.

El Análisis y Cartografía de la Vulnerabilidad a la Inseguridad Alimentaria en Bolivia de FAO/PMA (2002) concluye con que existe una alta concentración de los municipios vulnerables en el altiplano, parte de los valles y algunos municipios de Pando. Ormachea, E. (2010) resalta que los datos referidos al comportamiento de las importaciones de productos de origen agropecuario, permiten sostener que el país tiende a depender de una mayores alimentos importados.

El propósito del trabajo es evaluar las dimensiones(i.e. disponibilidad de alimentos, el consumo y utilización biológica, la estabilidad y la soberanía alimentaria) de la Seguridad Alimentaria (SA). Para lo cual se evalúa la disponibilidad de alimentos en términos de la diferencia entre la oferta y el requerimiento calórico.

Nutricionalmente se observan las diferencias entre los grupos de alimentos necesarios para una buena alimentación y su requerimiento óptimo. Ambas dentro de un marco temporal para mostrar la estabilidad de la SA en el tiempo y se analiza el componente importado para evaluar la soberanía.

El documento presenta la siguiente estructura: la siguiente sección revisa el marco conceptual y la forma en la que se cuantifica la SA, posteriormente se presenta la aproximación metodológica utilizada, a continuación se exponen los criterios utilizados para su operacionalización, seguido de los principales resultados y por último las conclusiones más pertinentes.

1 Encontrándose en las categorías 4-5. El índice denominado VAM, tiene 5 categorías. Dichas categorías son, de mayor a menor: altamente vulnerable (5), muy vulnerable (4), medianamente vulnerable (3), poco vulnerable (2) y no vulnerable (1). 


\section{2. ¿Qué es Seguridad Alimentaria?}

Dentro de la comunidad científica no existe un consenso sobre la definición de SA, debido a que la misma ha ido evolucionando en el transcurso del tiempo. El término aparece por primera vez a mediados de los setentas debido a la crisis de alimentos global ${ }^{2}$. El significado ha evolucionado significativamente durante las últimas décadas Haddinott, J. (1999) y desde entonces se han hecho varias redefiniciones de ésta.

Por lo que en esta sección se citaran las consideraciones más importantes y sus variaciones en función al tiempo, haciendo hincapié en que antiguamente se relacionaba la SA como un derecho que debería ser provisto por el Estado y en la actualidad se involucran agentes particulares para promoverla.

En los setentas la atención mundial se enfocó fundamentalmente en la oferta y almacenamiento de alimentos, buscando asegurar la disponibilidad y estabilidad de los precios de los alimentos básicos, en este sentido la Organización de las Naciones Unidas (ONU) define la SA como:

"la disponibilidad en todo momento de adecuados suministros mundiales de alimentos básicos para mantener de forma constante el consumo, sin que se vea afectado por las fluctuaciones de producción y de precios". Cumbre Mundial de la Alimentación celebrada en 1974.

"seguridad de suministros y situación de equilibrio entre oferta y demanda de alimentos básicos en el mercado internacional". Informe del Programa Mundial de Alimentos en 1979.

De la Vega, A. (2004) explica que en los años ochenta resultó evidente que la oferta no bastaba por sí sola para asegurar el acceso de la población a los alimentos ya que quedó demostrado que las hambrunas ocurren sin que exista déficit de alimentos a nivel mundial, por lo que se incluyó la importancia del acceso en una dinámica temporal. Debido a estas situaciones la SA pasa de ser entendida en términos de oferta a ser comprendida desde el punto de vista de la demanda ${ }^{3}$ :

"cuando todas las personas tienen en todo momento acceso físico y económico a los alimentos básicos que necesitan” (FAO 1983).

En relación a la calidad de la alimentación se introduce el concepto de alimentos sanos y nutritivos, con el que se enfatiza el hecho de que no solo es necesaria la existencia de los alimentos, si no que éstos deben ser nutritivos, el Banco Mundial (BM) plantea la siguiente definición:

"el acceso de todas los personas y en todo momento a suficientes alimentos para una vida activa y sana" Tomado del informe "Pobreza y Hambre" BM (1986)

A fines de los ochentas la FAO (1996) incorpora las preferencias, éstas deben ser interpretadas dentro del contexto de las diferentes tradiciones, culturas y creencias religiosas

2 En 1973 con motivo de la crisis energética se produjo la caída del dólar, seguida de una importante inflación que repercutió en una subida del precio de los insumos de producción agrícola. Ello trajo como consecuencia un retroceso en la producción agrícola.

3 Donde la demanda individual de bienes alimentarios puede ser abastecida de tres maneras: autoabastecimiento, comprándolos u obteniéndolos de la ayuda alimentaria. 
que frecuentemente se relacionan con las costumbres alimentarias de las comunidades ${ }^{4}$, esta aproximación conceptual se define como:

"La SA a nivel individual, familiar, nacional, regional y global se alcanza cuando todas las personas tienen en todo momento, accesofísico a suficientes alimentos inocuos, sanos y nutritivos que les permitan satisfacer sus necesidades y sus preferencias alimentarias para llevar una vida activa y sana", (FAO 1996).

Finalmente FAO (2001) hace referencia a las desigualdades de acceso dentro de un mismo hogar por motivos de discriminación de género hacia las mujeres u otros tipos de jerarquía familiar e incorpora el término social. Al ser ésta la última definición de SA, ampliamente aceptada, es la que es utilizada en la presente investigación, sin duda alguna, para llegar a esta se partió de las anteriores y se intentó corregir sus carencias. A su vez, esta definición fue desagregada en cinco dimensiones.

"Existe SA cuando todas las personas tienen en todo momento acceso físico, económico y social a alimentos, suficientes, inocuos y nutritivos para cubrir sus necesidades alimenticias y sus preferencias culturales en cuanto a los alimentos, a fin de llevar una vida sana y activa" FAO (2001).

\subsection{Dimensiones de la Seguridad Alimentaria}

Barrett (2002) señala que, como el concepto de SA es inherentemente inobservable, durante mucho tiempo se ha eludido una definición operacional precisa. Este autor identifica tres fases en el desarrollo del concepto de las cuales surgen las cinco dimensiones.

a) Para la primera dimensión, el énfasis recayó en el lado de la oferta, dando lugar a la importancia de la disponibilidad la cual según el Informe de políticas de la FAO (2006) es definida como la existencia de cantidades suficientes de alimentos de calidad adecuada $^{6}$ y suministrados a través de la producción del país y en caso extremo por importaciones o ayuda alimentaria externa.

b) Seguidamente se buscó abarcar la SA por el lado de la demanda, lo cual dio lugar al acceso a los alimentos que implica el acceso físico, económico y social, de los agentes a los recursos adecuados para adquirir alimentos apropiados ${ }^{7}$, garantizando la inocuidad de los alimentos por medio de un control de sanidad.

c) En la tercera fase se concibe a la SA dentro del marco de la elección intertemporal, abarcando la estabilidad, lo que implica el cumplimiento de las otras dimensiones de manera continua, estable y sin correr el riesgo de quedarse sin alimentos a consecuencia de crisis repentinas ${ }^{8}$, es decir; resolviendo las condiciones de inseguridad o acontecimientos cíclicos; como la IA estacional.

4 A sí mismo la última Conferencia Mundial de los Pueblos sobre Cambio Climático y la Defensa de los Derechos de la Madre Tierra (CMPCC) introduce el término de la soberanía alimentaria "implica el derecho de los pueblos a controlar sus propias semillas, tierras, agua y la producción de alimentos, garantizando, a través de una producción agroecológica, local y culturalmente apropiada, el acceso de los pueblos a alimentos suficientes, variados y nutritivos en complementación con la Madre Tierra y profundizando la producción autónoma de cada nación y pueblo" (CMPCC, 2010).

5 La definición actual de seguridad alimentaria habla de alimentos nutritivos para todos lo que hace que el término "seguridad alimentaria familiar" sea redundante y por tanto innecesario (FAO 1996).

6 Para considerar adecuados a los alimentos se requiere que sean culturalmente aceptables (incluy endo factores sociales como religiosos) y que se produzcan en forma sostenible tanto para el medio ambiente como para la sociedad.

7 Por el lado de la oferta implica el acceso a factores de producción. En economía los Factores de producción son K, T y L, acompañados de tecnología.

8 Se define crisis repentina a un shock que recibe una economía ante una externalidad; económica (incremento de precios, crisis internacionales), o climática (diluvios o sequías, entre otras). 
d) La dimensión de consumo y utilización biológica es tomada de la seguridad nutricional, Gros., y Schoengerber (2002) quienes explican que el marco conceptual para el análisis de la malnutrición ha sido desarrollado por $\mathrm{UNICEF}^{9}$ (1990) y está ligado a la seguridad nutricional, centrándose en el consumo de alimentos por unidad (familiar o individuo) y la forma en que los alimentos son utilizados por el organismo.

e) La última dimensión emerge de la vía campesina ${ }^{10}$ comprendiendo la soberanía alimentaria $^{11}$, es considerada como el derecho de los pueblos, naciones o uniones de países a definir sus políticas agrícolas y de alimentos, sin ningún dumping ${ }^{12}$ frente a otros países.

\section{3. ¿Cómo se "mide" la Seguridad Alimentaria?}

Giraldo D., Betancur, M. y Arango, S., (2011) desagregan las metodologías utilizadas en índices (Véase, Anexo 1). A nivel agregado provienen de dos tipos de modelos: proyección de tendencia y comercio mundial. Los primeros pronostican la brecha entre el consumo y la producción alimentaria, suponiendo que los precios relativos son constantes en el tiempo. La segunda línea permite evaluar la interacción entre varios países en relación al comercio, encontrando precios y cantidades. La Tabla 1 muestra trabajos que utilizan estas metodologías.

Tabla 1

Características de los modelos más reconocidos

\begin{tabular}{|c|c|c|c|}
\hline Nombre & Metodología & Alcance & Fuente \\
\hline $\begin{array}{l}\text { International } \\
\text { Model for Policy } \\
\text { Analy sis of } \\
\text { Agricultural } \\
\text { Commodities and } \\
\text { Trade IFPRI }\end{array}$ & $\begin{array}{l}\text { Modelo de } \\
\text { equilibrio } \\
\text { parcial }\end{array}$ & $\begin{array}{l}\text { Ofrece una metodología para el } \\
\text { análisis de líneas de referencia y } \\
\text { escenarios alternativos de la } \\
\text { demanda global de alimentos, } \\
\text { oferta, comercio, ingreso, población }\end{array}$ & $\begin{array}{l}\text { Rosegrant, M.W., Ringler, C., M sangi, } \\
\text { S., Cline, SA y Sulser, TB (2005). } \\
\text { International Model for Policy Analy sis } \\
\text { of Agricultural Commodities and Trade } \\
\text { (IMPAC-WATER): Model Description. }\end{array}$ \\
\hline $\begin{array}{l}\text { Food Security } \\
\text { Assessment } \\
\text { USDA }\end{array}$ & $\begin{array}{l}\text { Modelo de } \\
\text { equilibrio } \\
\text { parcial }\end{array}$ & $\begin{array}{l}\text { Evalúa la SA de un país mediante la } \\
\text { brecha existente entre la proyección } \\
\text { del consumo doméstico alimentario } \\
\text { y el requerido en } 70 \text { países. }\end{array}$ & $\begin{array}{l}\text { Shapouri, S. y Rosen, S. (2005) Food } \\
\text { Security Assessent. Electronic Outlook } \\
\text { Report fron the Economic Research } \\
\text { Service. GFA-16. }\end{array}$ \\
\hline $\begin{array}{l}\text { Policy Dialogue } \\
\text { Model. A Water } \\
\text { and Food Security } \\
\text { Planning Tool }\end{array}$ & $\begin{array}{l}\text { Modelo de } \\
\text { proyección de } \\
\text { Tendencia }\end{array}$ & $\begin{array}{l}\text { Determina la demanda de agua en } \\
2025 \text { y encuentra los } \\
\text { requerimientos alimentarios como } \\
\text { resultado del crecimiento de la } \\
\text { población y los cambios en la dieta. }\end{array}$ & $\begin{array}{l}\text { Seckler, D., Amarasinghe, U., Molden, } \\
\text { D., de Silva, R., Barker, R., (1998). } \\
\text { World water demand and supply, } 1990 \\
\text { to 2025: scenarios and issues. Research } \\
\text { Report } N^{\circ} 19, \text { International }\end{array}$ \\
\hline World Food FAO & $\begin{array}{l}\text { Modelo de } \\
\text { equilibrio } \\
\text { general }\end{array}$ & $\begin{array}{l}\text { Provee un marco para proyectar la } \\
\text { disponibilidad, acceso y flujo neto } \\
\text { alimentario entre } 150 \text { países. }\end{array}$ & $\begin{array}{l}\text { Water Management Institute(IWMI), } \\
\text { Colombo, Sri Lanka. FAO. (1993). The } \\
\text { World Food Model-Model } \\
\text { Specifications ESC/M/93/1. }\end{array}$ \\
\hline
\end{tabular}

Fuente: Extraído de Análisis Metodológico para la Modelación de la Seguridad Alimentaria a nivel nacional.

$9 \quad$ Sobre la base del Marco conceptual de UNICEF, el Instituto Internacional de Investigación sobre Políticas Alimentarias (IFPRI-1995) propuso que "la seguridad nutricional se puede definir como un estado nutricional adecuado en términos de proteínas, energía, vitaminas y minerales para todos los miembros de la unidad familiar en todo momento".

10 La Vía Campesina (movimiento internacional) es una coalición de 148 organizaciones en 69 países del mundo defendiendo una agricultura familiar y sustentable. Esta coalición lanzó el concepto de soberanía alimentaria.

11 La última Conferencia Mundial de los Pueblos sobre Cambio Climático y la Defensa de los Derechos de la Madre Tierra (CMPCC) ha indicado que la soberanía alimentaria "implica el derecho de los pueblos a controlar sus propias semillas, tierras, agua y la producción de alimentos, garantizando, a través de una producción agroecológica, local y culturalmente apropiada, el acceso de los pueblos a alimentos suficientes, variados y nutritivos en complementación con la Madre Tierra y profundizando la producción autónoma de cada nación y pueblo" (CMPCC, 2010).

12 Se entiende dumping como la práctica en donde una empresa establece un precio inferior para los bienes exportados que para los costos de producción que tiene la empresa desde el país a donde se importan esos bienes, sacando de competencia a la empresa local. 
Para el propósito de esta investigación se sigue la línea de los modelos de proyección de tendencia, debido a que se pronostica la brecha entre el consumo, en este caso el requerimiento óptimo y la oferta interna ${ }^{13}$ que incluye la producción del país. En la siguiente sección se presenta la aproximación metodológica que se utiliza en este documento.

\section{Aproximación Metodológica}

Debido a que resulta difícil hacer un estudio que abarque las cinco dimensiones de la SA y a su vez cumpla con los tres determinantes de la SN. La presente metodología abarcará las dimensiones de consumo y utilización biológica, disponibilidad, estabilidad y soberanía entendida como autarquía ${ }^{14}$, dejando de lado la dimensión de acceso a los alimentos y los determinantes de la SN.

a) Para corroborar la disponibilidad, se observa la participación del componente importado dentro de la oferta alimentaria.

b) Bajo el supuesto de una distribución equitativa, se trabaja con una oferta per cápita, lo cual impide que se analice la dimensión de acceso a los alimentos.

c) Para evaluar la estabilidad, el análisis realizado es de carácter temporal, dentro de un periodo considerable (1990-2012).

d) Dentro de este documento se entenderá soberanía como autarquía, ya que al auto abastecerse, la producción boliviana es soberana sobre la importada e implica que se consumen alimentos tradicionales.

e) Para que se cumpla la dimensión de consumo y utilización biológica la oferta alimentaria debe abastecer los requerimientos nutricionales mínimos de la población.

Para dicho propósito, se calcula la oferta alimentaria interna y el requerimiento que demanda una población bien alimentada. La diferencia entre la oferta y demanda generan la brecha que bajo determinados criterios explicarán si el país cumple cuatro de las cinco dimensiones de la SA.

Por un lado la oferta se compone de alimentos producidos internamente e importados, la producción se destina a la exportación y al consumo interno. Las importaciones proceden de donaciones, comercio por vía legal e ilegal. Toda esta oferta alimentaria crea una cesta en la que la composición porcentual define la relevancia del sector externo e interno dentro de la economía para la SA del país.

La demanda de alimentos es afectada por el crecimiento poblacional. Sectorialmente, sea la población urbana o rural, la cantidad de energía que requerirá depende de las actividades que realice y el clima en el que la población habite. Esta demanda calculada siguiendo criterios de consumo meritorio, se representa por una línea nutricional ${ }^{15}$. La cual es calculada a partir de los requerimientos energéticos de un agente promedio, que se explicará a detalle a continuación.

13 Oferta interna: que definimos como la cantidad de bienes alimentarios que se encuentra dentro del mercado nacional, sin hacer referencia al origen de la producción.

14 Sistema económico según el cual una nación debe ser capaz de abastecerse a sí misma y subvenir a todas sus necesidades con un mínimo de intercambios comerciales con el exterior y rechazando los capitales extranjeros. Es sinónimo de economía cerrada al comercio exterior o al mercado global, lo que conlleva establecer restricciones al intercambio entre individuos de distintos sitios.

15 Línea nutricional: se define como las cantidades mínimas necesarias de un producto alimentario para el sostenimiento de las funciones corporales del organismo humano dirigidas hacia una salud y rendimiento óptimos. Dicha línea se encuentra medida a precios constantes. 


\subsection{Línea Alimentaria Nutricional}

Según Elmadfa., Leitzmann., y Ernaehrung des Menschen (1990) el requerimiento de un nutriente se define como la cantidad necesaria para el sostenimiento de las funciones corporales del organismo humano dirigidas hacia una salud y rendimiento óptimos. Los requerimientos nutricionales del ser humano tienen tres componentes: el requerimiento basal; el requerimiento adicional (crecimiento, gestación, lactancia o actividad física) y la adición de seguridad para considerar pérdidas de nutrientes por manipulación y procesamiento.

La línea alimentaria nutricional es el requerimiento mínimo de un bien alimentario a nivel per cápita ponderado a precios constantes. Para cuantificarla se utilizará el método de ingesta de calorías Food Energy Intake $(\mathrm{FEI})^{16}$.La FAO (2008) utiliza este método para su actualización de las necesidades energéticas mínimas. Dentro de este método se trabaja bajo el supuesto de individuos saludables, es decir que no se toman requerimientos adicionales para condiciones de sub nutrición.

\subsubsection{La Tasa de Metabolismo Basal}

La Tasa de Metabolismo Basal (TMB), es la medida de la energía necesaria en calorías para mantener el funcionamiento del cuerpo en reposo ${ }^{17}$, se mide en condiciones estandarizadas: 12-14 horas después de una comida y en un ambiente térmico neutro ${ }^{18}$. La FAO/OMS (2004) utiliza las ecuaciones predictivas desarrolladas por Dubois en 1916. Autores como Ismail, N., Chee, S., Roslee, R. y Zawiah, H. (1998) replicaron la metodología para Malasia. Estas ecuaciones están basadas en variables como: edad, género y peso (Véase Tabla 2).

Tabla 2

Valores promedios de las ecuaciones utilizadas para el cálculo de las necesidades de Energía

\begin{tabular}{ccc} 
ADULTOS & Edad en años & $\mathrm{kcal} / \mathrm{d}$ \\
\hline \hline \multirow{2}{*}{ HOMBRES } & $18.0-29.99$ & $15.057 \mathrm{~kg}+692.2$ \\
& $30.0-59.99$ & $11.472 \mathrm{~kg}+873.1$ \\
& $60 \mathrm{y}$ más & $11.711 \mathrm{~kg}+587.7$ \\
\hline \multirow{2}{*}{ MUJERES } & $18.0-29.99$ & $14.818 \mathrm{~kg}+486.6$ \\
& $30.0-59.99$ & $8.126 \mathrm{~kg}+845.6$ \\
& 60 y más & $9.082 \mathrm{~kg}+658.5$ \\
\hline
\end{tabular}

Fuente: FAO/OMS (2004)

16 Esta metodología supone la definición de una ingesta mínima de alimentos por parte de un individuo a fin de que pueda llevar a cabo una vida decente. Con esta acepción, aquellos individuos que no puedan asumir el coste de la FEI son considerados pobres. El concepto de pobreza que se deriva se construye exclusivamente a partir de la alimentación como necesidad básica. Será pues especialmente precisa en realidades subdesarrolladas en las cuales una fracción significativa del presupuesto se asigne a este concepto.

En países más desarrollados, la proporción destinada a alimentación será menor y, por tanto, será necesario completar la cesta con otros bienes no alimenticios.Tal y como plantea Deaton (1997), esta aproximación a los estándares de vida a partir del consumo de una cesta de alimentación adecuada entra en conflicto con una visión meramente economicista.

17 Reposo corporal y mental. Esta energía mínima es utilizada por la célula en las reacciones químicas intracelulares necesarias para la realización de funciones metabólicas esenciales, como es el caso de la respiración.

18 El gasto energético tiene 3 fuentes donde alrededor del 60-70\% se da por la TMB, del 20-30\% se genera por el Nivel de Actividad Física (NAF) y el saldo por la acción termogénica de los alimentos consumidos. 
Se utilizaron las ecuaciones establecidas por Schofield (1985) en las que se revisaron aproximadamente 11.000 mediciones $^{19}$ de la TMB pata inferir ecuaciones de predicción para hombres y mujeres. Estas ecuaciones luego fueron adoptadas para su uso en la FAO-OMSUNU (1985).

$$
I M C=\frac{W}{H^{2}}
$$

Donde:

$$
\begin{aligned}
& I M C=\text { Índice de Masa Corporal } \\
& W=\text { Peso en } \mathrm{kg} \\
& H=\text { altura en } m \\
& \quad \text { Despejando W se tiene: }
\end{aligned}
$$

$$
W=I M C * H^{2}
$$

Introduciendo el valor $\mathrm{W}$ en las ecuaciones de predicción, se encuentra la TMB:

Donde:

$$
T M B=\alpha+\beta W+\varepsilon
$$

$T M B=$ Tasa de metabolismo basal

$W=$ Peso corporal óptimo en $\mathrm{Kg}$

$\varepsilon=$ error de estándar

\subsubsection{Nivel de Actividad Física}

El Nivel de Actividad Física (NAF) es un múltiplo de la TMB y la cantidad de energía (en kilocalorías) que una persona necesita para la línea de base de funcionamiento del cuerpo en reposo ${ }^{20}$. En función al área en el que viva la población (sea rural o urbano) el tiempo dedicado a cada actividad varía y por lo tanto el NAF cambia (Véase, Anexo 2).

$$
N A F=\delta T M B
$$

Donde:

$\delta=$ proporción de la TMB

\subsubsection{Gasto Energético}

Las mediciones de gasto de energía (5) se expresan como unidades de energía termoquímica (kilocalorías $(\mathrm{kcal}))^{21}$, es decir; la energía total en kilocalorías que se necesita para satisfacer las necesidades diarias de energía de cada agente en una determinada edad o género (o de toda la población). La cantidad de energía que requiera la población se encuentra dentro de los parámetros estimados internacionalmente ${ }^{22}$.

19 Para las mediciones de las TMB, se utilizó el método de agua doblemente marcada, así como también un monitoreo de la frecuencia cardiaca.

20 Se indica el nivel de energía necesaria tanto para el funcionamiento línea de base y el resto de la actividad física llevada a cabo por el agente, además de la absorción, distribución y almacenamiento de energía de los alimentos.

21 La unidad de energía, kcal, es la cantidad de energía necesaria para elevar la temperatura de $1 \mathrm{~g}$ de agua por $1^{\circ} \mathrm{C}$. Los múltiplos de mil kilocalorías $((\mathrm{kcal}))$ se utilizan en la nutrición humana.

22 Los requerimientos de energía contenidos en esta investigación contemplan el supuesto de tener individuos "sanos", no se contemplan energía y nutrientes adicionales para enfrentar condiciones de desnutrición, enfermedades, infecciones y otras patologías. Según la Organización Mundial de la Salud la ingesta en Bolivia fluctúa entre 2000 y 2300 [kcal/día]. 


$$
e=T M B+N A F
$$

Donde:

$e=$ gasto de energía

Para la creación de la cesta el requerimiento energético utilizado es mayor al gasto energético, debido a que se considera el desperdicio y los servicios de comida, entre otros.

Donde:

$$
E=e+\gamma e
$$

$E=$ requerimiento energético

$e=$ gasto de energía

$\gamma=$ proporción destinada al desperdicio

Aun siendo posible formular una dieta que cumpla con los requerimientos nutricionales mínimos, no hay que olvidar que los destinatarios de esa dieta son personas y que los agentes comen alimentos, no nutrientes. La línea alimentaria nutricional es fijada en función de requerimientos alimenticios mínimos.

Para el nivel de energía requerida a nivel per cápita, se realiza la conversión del requerimiento energético ${ }^{23}$ en alguna unidad de medida, por ejemplo gramos, kilogramos, etc, según sus respectivas equivalencias, es decir kcal a gramos según cada producto alimentario. Dado que para la oferta se trabaja para el periodo de 1990-2012, el requerimiento también tendrá una frecuencia anual, ecuación (7) y será ponderado en precios constantes para que sea contrastado con la oferta interna ecuación (8):

$$
\begin{aligned}
& \Re=\text { requerimiento anual en }[u m] \\
& \Re=u m * 365 \text { dias }
\end{aligned}
$$

Habiendo encontrado el requerimiento, éste es ponderado a precios constantes, para poder ser comparado con la oferta interna, formando así la línea alimentaria nutricional:

$$
L A N=\mathscr{R} * P
$$

Donde:

$$
\begin{aligned}
& L A N=\text { Linea Alimentaria Nutricional } \\
& R=\text { requerimiento óptimo en cantidad } \\
& P=\text { Precios }
\end{aligned}
$$

\subsection{Oferta}

Sea la oferta interna per cápita ecuación (9), la cual debe abastecer el consumo mínimo per cápita requerido por la población, definido por la línea nutricional.

$$
O_{t}=\frac{P_{t}-X_{t}+M_{t}+\varepsilon_{t}}{N_{t}}
$$

Donde:

$O=$ oferta de los bienes alimentarios

$P=$ producción interna de bienes alimentarios, destinados al consumo

${ }^{23}$ Para dichas conversiones se utilizaron las tablas aprobadas por el Colegio Nacional Nutricionistas (Bolivia). 
$X=$ exportación de bienes alimentarios

$M=$ importación de bienes alimentarios

$\varepsilon=$ error

$N=$ Población

\subsection{Equilibrios}

Los posibles resultados del trabajo mostraran, por un lado la situación del agente promedio respecto a su ingesta calórica y por otro lado, si la fuente de dicha energía cumple requerimientos nutricionales. A la luz de ambos instrumentos en función a la fuente de la oferta, sea de origen propio, importado o donado, se concluirá el estado de lasdimensiones de la seguridad alimentaria del país a partir de considerar la brecha que es la diferencia existente entre la línea alimentaria nutricional (LAN) y la oferta en la sucesión de varios periodos $\left(O_{t}\right)$.

$$
L A N=O_{t}
$$

\subsubsection{Brecha Calórica}

De manera general la ingesta de alimentos puede abastecer, sobrepasar o ser insuficiente dado el requerimiento calórico. La construcción de estos escenarios requiere el gasto de energía de los agentes ecuación (6) y la oferta ponderada en kilocalorías netas, de esta manera se obtiene los posibles escenarios siguientes:

a) Con excedente: la oferta abastece el requerimiento con una brecha positiva $O>e$.

b) Suficiente: situación de equilibrio en la que la oferta abastece con el requerimiento $O=e$.

c) Insuficiente: la oferta es insuficiente para el requerimiento de la población $O<e$

\subsubsection{Brechas Nutricionales}

Para encontrar las brechas a nivel nutricional, se plantea un análisis para cada grupo de alimento utilizando la versión del "Plato de Harvard", el cual se ilustra en la Figura 1 que representa un plato óptimo de ingesta de alimentos, que distribuye los alimentos en tres subgrupos; carbohidratos, proteínas y frutas y verduras. Dentro de cada grupo de alimento se encontrarán distintas brechas al comparar el requerimiento mínimo de dicho producto alimentario con la oferta, encontrando así los distintos posibles resultados, explicados a continuación.

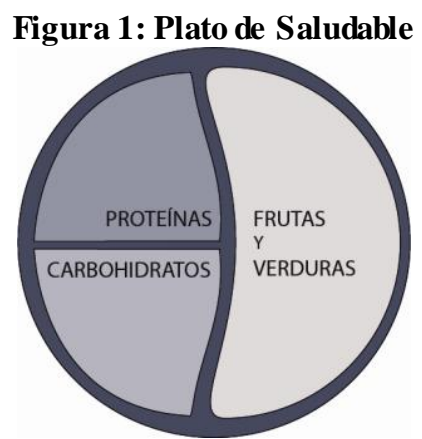

Fuente: Elaboración de los autores en base al logo del Plato de Harvard. 


\section{$5 \quad$ Criterios de Evaluación}

\begin{tabular}{|c|c|c|c|}
\hline DIMENSIONES & CUMPLE & NO CUMPLE & $\begin{array}{c}\text { JUSTIFICACION } \\
\end{array}$ \\
\hline Disponibilidad & $L A N<0$ & $L A N>0$ & $\begin{array}{l}\text { La oferta supera a la línea alimentaria, lo cual implica que } \\
\text { se abastece el mercado interno. Puede haber un exceso de } \\
\text { oferta interna, además el país puede exportar el saldo. }\end{array}$ \\
\hline Soberanía & $\begin{array}{l}L A N=P \\
L A N<P\end{array}$ & $L A N>P$ & $\begin{array}{l}\text { La producción del país es destinada para el consumo } \\
\text { interno, sin comercio exterior } L A N=P \text {. El comercio } \\
\text { exterior puede ser favorable o no incidiendo en la } \\
\text { soberanía alimentaria. } \\
\text { No se contará con soberanía alimentaria si el comercio } \\
\text { exterior se da por razones comerciales }\left(\overline{L A}>O_{t}\right) \text {. } \\
\text { Se contará con soberanía alimentaria si el comercio existe } \\
\text { debido a ventajas comparativas } \text { le }^{24} \text { en la producción de } \\
\text { algunos alimentos. Por lo que la razón del comercio es } \\
\text { para la diversidad de la oferta }\left(\overline{L A}<O_{t}\right) \text {. }\end{array}$ \\
\hline Estabilidad & $L A N_{t} \leq O_{t}$ & $L A N_{t}>O_{t}$ & $\begin{array}{l}\text { La estabilidad implica disponibilidad en la sucesión de } \\
\text { varios periodos. }\end{array}$ \\
\hline $\begin{array}{l}\text { Consumo } \\
\text { Utilización } \\
\text { Biológica }\end{array}$ & $L A N \leq O$ & $L A N>0$ & $\begin{array}{l}\text { Para el cumplimiento de dicha dimensión la oferta deberá } \\
\text { abastecer el requerimiento mínimo nutricional de cada } \\
\text { grupo de alimento. Para lo cual se revisarán las brechas } \\
\text { nutricionales. }\end{array}$ \\
\hline
\end{tabular}

\section{Criterios para la Operacionalización}

Para el propósito de esta investigación lo más importante es definir la línea nutricional para lo cual se utilizan criterios nutricionales. En base a las cestas se define una línea nutricional para cada producto, para hacer el cálculo de éste se inicia calculando el requerimiento de energía de un boliviano promedio. Dicho agente destina su energía en función a su tasa metabólica basal (TMB), el cual es el gasto energético de línea base que requerimiento y el gasto se incrementa en función al nivel de actividad física (NAF).

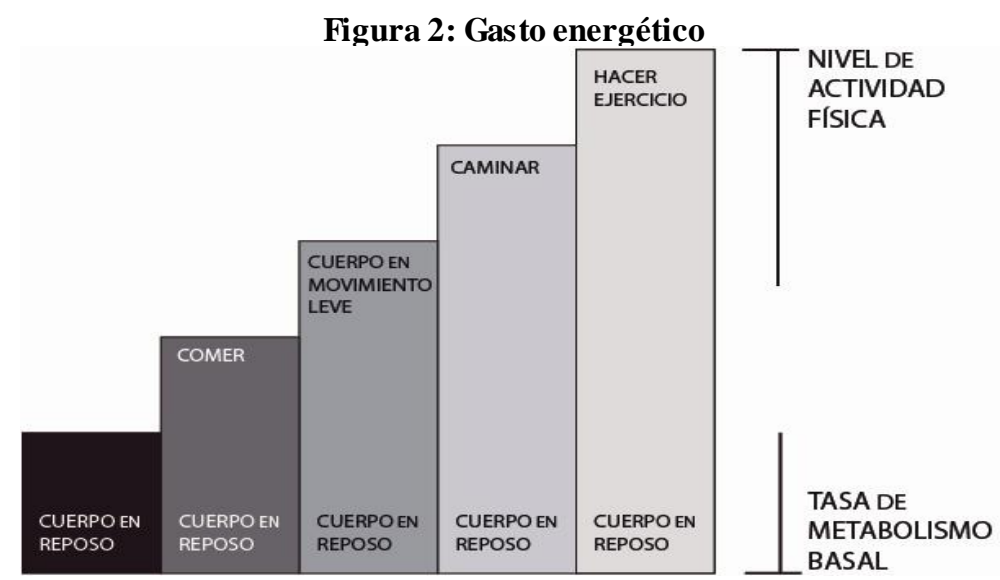

Fuente: Elaboración de los autores.

${ }^{24}$ El modelo de la ventaja comparativa es uno de los conceptos básicos que fundamenta la teoría del comercio internacional y muestra que los países tienden a especializarse en la producción y exportación de aquellos bienes que fabrican con un coste relativamente más bajo respecto al resto del mundo, en los que son comparativamente más eficientes que los demás y que tenderán a importar los bienes en los que son más ineficaces y que por tanto producen con unos costes comparativamente más altos que el resto del mundo. 


\subsection{Cestas}

Dentro de la literatura de la buena alimentación, el plato de Harvard y la versión de MyPlate de Estados Unidos para la composición de alimentos, son los estudios más novedosos, ambos son introducidos en esta investigación. Para poder hacer un análisis a nivel nutricional se tomaron tres grupos de alimentos: carbohidratos, frutas y verduras y proteínas, grupos de productos que continúan desagregándose ${ }^{25}$.

$\mathrm{Si}$ bien los requerimientos nutricionales, entendidos como la participación de cada grupo de alimento dentro de una dieta, no varían en gran medida en el tiempo, la evidencia empírica muestra que la ingesta energética, es decir, la cantidad de calorías que se requieren dado un periodo de tiempo puede variar; por lo que para el presente trabajo se realizarán dos mediciones, específicamente para los años: 2001 y 2012, para el cálculo de los requerimientos energéticos (e) y con ellos inferir los requerimientos nutricionales $(\mathfrak{R})$.

\subsection{Línea Nutricional}

Para facilitar la aplicación de esta metodología FAO y OMS desarrollaron una herramienta de aplicación estandarizada para el cálculo de los requerimientos calóricos denominada Population Energy Requirement ${ }^{26}$ (POPER).

\subsubsection{Tasa de Metabolismo Basal}

Para las ecuaciones (1) y (2) no existen datos nacionales sobre pesos y alturas. Izquierdo, S. (2014) explica que los últimos estudios antropométricos de la evaluación del crecimiento de niños de 0 a 5 años y de 5 a 19 años, demuestran que un niño alimentado con el seno materno por lo menos hasta los seis meses y con adecuada ingesta complementaria en cualquier parte del mundo tendrá el mismo crecimiento.

Los resultados del Censo de Talla realizado en 1989-1990 en escolares, confirmaron un alto nivel de retardo de crecimiento (35\%), siendo los varones de 6 a 9 años los más afectados. La situación fue aún más grave en el área rural (40\%) y en 18 provincias de los departamentos de Potosí, Cochabamba y Chuquisaca, caracterizadas por altas tasas de pobreza y de analfabetismo, falta de infraestructura y de servicios básicos y poca o ninguna potencialidad para la producción agropecuaria.

La única información acerca de los adolescentes (12-17 años) fue recolectada en 1993 en las escuelas de la ciudad de La Paz y evidenció 19\% de retardo de crecimiento. En la ENDSA de 1998 se indica que la talla en madres de 15-19 años con niños menores de 3 años es de $151 \mathrm{~cm}$ y el Índice de Masa Corporal promedio es de $25,3 \mathrm{~kg} / \mathrm{m} 2$, ligeramente mayor a lo encontrado en 1994. Con referencia a los adultos no existe información a nivel nacional.

A sí mismo concluye que respecto a la talla baja de la población boliviana, es principalmente debida a la desnutrición crónica que le afecta ${ }^{27}$. Por lo que, Izquierdo (2014) recomienda utilizar pesos $(\mathrm{W})$ y alturas $(\mathrm{H})$ óptimas según edades, en base a las cuales se

\footnotetext{
25 Se desagregan según el tipo de clasificador que el INE utiliza, hasta llegar a productos precisos.

Janice Albert (Oficial de nutrición de FAO) expresa que este software se creó en una sola versión, y en la actualidad aún se la utiliza. Vialle Delle Terme di Caracala 00153 Roma, Italia.

27 Lo cual corrobora mediante la aplicación del software para la Evaluación Antropométrica Individual y poblacional de la OMS (ANTRO y ANTROPLUS).
} 
calculó el IMC, y con los resultados, según las ecuaciones de predicción (3) se obtuvieron las TMB.

Por lo que para esta investigación se utilizaron los valores recomendados por la OMS (2009) para países en vías al desarrollo. De los cuales las alturas mencionadas se utilizan para calcular los pesos corporales medios específicos de la edad provienen de las curvas de crecimiento previstas en el 1985 James y manual Schofield. Estas curvas de crecimiento se utilizan en la actualidad, la OMS ha desarrollado una amplia literatura sobre el tema.

Las alturas mencionadas se utilizan para calcular los pesos corporales promedio específicas por edad provienen de las curvas de crecimiento proporcionadas en 1985 por James y Schofield. La edad y el peso corporal específicos del sexo vienen de un perfil de alturas derivados mediante la agrupación de países similares, que han sido convertidos a "óptimas" pesos corporales de altura dada por la FAO (2004).

\subsubsection{Nivel de Actividad Física}

Al ser un múltiplo de la TMB que una persona necesita sobre la línea de base de energía para funcionamiento del cuerpo en reposo ${ }^{28}$. En función al área en el que viva la población existen distintos costos establecidos, por lo que la proporción de la TMB $(\delta)$ oscila dentro de un rango de 0,10-1,00 tanto para el área rural como urbano. Expertos de la FAO recomiendan que para países en vías al desarrollo se mantenga entre 0,10 y 0,50 (se recomienda utilizar 0,10 área urbana y 0,50 área rural) sobre el nivel de gasto energético de línea base, es decir de la TMB.

Es de esperar que en el área rural el nivel de actividad física (NAF) sea mayor porque se realizan actividades agropecuarias, agroindustriales, extractivas y de silvicultura. En un año agrícola las épocas de siembra y cosecha incrementan la jornada laboral entre dos y cuatro horas más que en el área urbana además en el área rural el trabajo manual es mayor, sobre todo en unidades campesinas no industrializadas.

Tabla 3

Asignación del Nivel de Actividad Física por piso Geográfico

\begin{tabular}{|c|c|c|c|c|c|c|}
\hline \multirow{2}{*}{$\begin{array}{l}\text { DEPARTAMENTOS } \\
\text { ÁREA }\end{array}$} & \multicolumn{2}{|c|}{ La Paz, Oruro y Potosí } & \multicolumn{2}{|c|}{$\begin{array}{l}\text { Cochabamba, Chuquisaca y } \\
\text { Tarija }\end{array}$} & \multicolumn{2}{|c|}{$\begin{array}{l}\text { Pando, Beni y } \\
\text { Santa Cruz }\end{array}$} \\
\hline & Urbano & Rural & Urbano & Rural & Urbano & Rural \\
\hline NAF & 0,25 & 0,45 & 0,20 & 0,40 & 0,15 & 0,35 \\
\hline $\begin{array}{l}\text { CARÁCTERÍSTICA } \\
\text { DE LA REGIÓN }\end{array}$ & \multicolumn{2}{|c|}{$\begin{array}{l}\text { Un clima frío implica } \\
\text { mayor gasto de energía } \\
\text { para mantener el cuerpo a } \\
\text { temperatura corporal } \\
\text { normal. }\end{array}$} & \multicolumn{2}{|c|}{$\begin{array}{l}\text { En climas templados dis minuye el } \\
\text { gasto energético para mantener la } \\
\text { temperatura corporal. El } \\
\text { movimiento y actividad física ya } \\
\text { implican mantener una } \\
\text { temperatura corporal normal. }\end{array}$} & \multicolumn{2}{|c|}{$\begin{array}{l}\text { Los climas cálidos requieren } \\
\text { de aún menor gasto } \\
\text { energético para mantener la } \\
\text { temperatura corporal. Un } \\
\text { cuerpo en estado de reposo, } \\
\text { se aclimata sin requerir de un } \\
\text { mayor gas to energético. }\end{array}$} \\
\hline PRODUCCIÓN & \multicolumn{2}{|c|}{$\begin{array}{l}\text { La producción agrícola } \\
\text { realizada en el altiplano } \\
\text { requiere de mayor actividad } \\
\text { física a diferencia de otras } \\
\text { actividades agrícolas. }\end{array}$} & \multicolumn{2}{|c|}{$\begin{array}{l}\text { El trabajo agrícola requiere menor } \\
\text { esfuerzo tanto por el tipo de } \\
\text { productos, como por elclima } \\
\text { propio del lugar. }\end{array}$} & \multicolumn{2}{|c|}{$\begin{array}{l}\text { El clima favorece a la } \\
\text { vegetación y hace que la } \\
\text { producción sea más fácil. Así } \\
\text { mis mo el Oriente del país } \\
\text { tiene la característica de ser } \\
\text { más industrializado. } \\
\end{array}$} \\
\hline
\end{tabular}

Fuente:Elaboración de los autores.

28 Se indica el nivel de energía necesaria tanto para el funcionamiento línea de base y el resto de la actividad física llevada a cabo por el agente, además de la absorción, distribución y almacenamiento de energía de los alimentos. 
Por lo que para asignar el NAF y obtener el gasto energético, como indica la Tabla 3, se tomaron en cuenta las características de cada región y sus necesidades por piso geográfico. Para todo el país se utilizaran los valores medios del NAF obtenidos de los distintos pisos geográficos, siendo éstos de 0,15 y 0,40 urbano y rural respectivamente. Utilizar distintos niveles de actividad física, diferenciándolos por piso geográfico es importante, ya que el requerimiento energético varía más en función al nivel de actividad física que en función a la talla real de la población ${ }^{29}$.

\subsubsection{Gasto Energético}

Al gasto energético obtenido según la ecuación (6), se le agrega la proporción destinada al desperdicio, el cual incluye, cáscaras, huesos y servicios de comida entre otros. Este gasto energético es de 0,25 al ser el recomendado ${ }^{30}$ para países en vías al desarrollo ya que se espera que en estos países el desperdicio represente lo mínimo. Para obtener la conversión del requerimiento energético a gr, se tiene:

$$
\text { E kcal } * \frac{100 \mathrm{gr}}{E^{r} \text { kcal }}=\text { energía en } \mathrm{gr}
$$

El valor estimado por la ecuación (10) posteriormente será expresado en toneladas métricas haciendo conversiones ${ }^{31}$, debido a que el Instituto Nacional de Estadísticas (INE) las utiliza como unidad de medida, para las Matrices Insumo-Producto, lo cual se explicará al analizar la oferta.

\subsection{Oferta}

Siguiendo la metodología del Instituto Nacional de Estadística(s/f) para la cuantificación de la oferta dada la ecuación (2) se utilizaron de las Matrices InsumoProducto $^{32}$, desde 1990 hasta 2012. Debido a que al hacerse el cambio de base dentro de los clasificadores hay variaciones estadísticas quedando una ruptura en el periodo 1990-2005 y 2006-2012.Al usar las MI-P se utilizaron datos agregados, quedando la serie completa y coherente.

Se los desagregó revisando la manera en la que se han agregado, por lo que teniendo los datos agregados, se los pudo desagregar hasta producto. Para lo que se utilizaron varios clasificadores, así mismo se eliminó la parte del producto destinada a consumo intermedio, como la producción agrícola que no tiene aporte nutricional ${ }^{33}$.

29 El nivel de actividad física puede representar un 25-40\% del total del requerimiento energético.

30 El desperdicio generado por el proceso de la preparación de alimentos y el de desechos como huesos, cáscaras y otros, no ha sido medido en el país es una aproximación calculada por expertos de la FAO. Sin embargo para tener una idea el desperdicio cuantificado en EEUU bordea el $50 \%$.

$31 \quad T M=$ Toneladas métricas diarias necesarias

$$
\frac{\mathrm{gr}}{1000 \mathrm{~kg}} * \frac{\mathrm{kg}}{1000 \mathrm{TM}}
$$

32 Para la elaboración de las Matrices Insumo Producto se cuenta con resultados estadísticos provenientes de censos, encuestas y registros administrativos; obtenidas de fuentes estadísticas muy diferentes: encuestas industriales, encuestas de gasto de los hogares, encuestas de inversión, estadísticas de comercio exterior, etc. Las cuales son sujetas a los errores e insuficiencias propias de esta clase de eventos en medios como el nuestro. Las Matrices Insumo Producto tienen un alto grado de agregación, para la cual se utilizan todos los clasificadores de cuentas nacionales, por lo que es información completa.

33 A la producción se le descontó: fibras, café y cacao, forrajes, flores, servicios prestados a los PANI y mejora de tierras y desarrollo de plantaciones del grupo productos agrícolas industriales. Como ser la producción de: tabaco en rama, fibra de algodón, hojas de té, servicios prestados a PAI, mejora de tierras y desarrollo de plantaciones del grupo productos 
Al utilizar las MI-P se obtuvieron los datos de las importaciones, exportaciones y el sub-registro. Para poder analizar la dependencia al sector externo. El tratamiento para cada año se realizó de manera individual, revisando los datos de cuentas nacionales del INE considerados datos no públicos para lo cual se trabajó en coordinación con dicho departamento estadístico. Por lo que a grandes a rasgos lo que se hizo fue tomar la producción interna $(\mathrm{P})$ destina para el consumo $^{34}$, de la cual se excluyen las exportaciones $(\mathrm{X})$ se añaden las importaciones (M) y el sub-registro ${ }^{35}$.

Siguiendo la metodología de la elaboración de las Matrices Insumo-Producto, para estimar el valor monetario del bien alimentario se utiliza el valor de la producción debido a que las unidades de medida para los productos son: tonelada, kilo fino, barril, metro cúbico, entre otros y éstos no son aditivos. Es decir, que para agregarlos es necesario encontrar una unidad de medida común.

Donde:

$$
V=Q x P
$$

$V=$ Valor monetario del bien alimentario
$Q=$ Cantidad del bien analizado
$P=$ Precios constantes de 1990

Las cantidades se miden en magnitudes físicas y se expresan de acuerdo al número de unidades producidas o según unidades de medida (longitud, peso, entre otros). El precio es el monto de dinero entregado por cada unidad de un producto, por lo que todas las variables a usar en el modelo se miden a precios constantes.

\section{Principales Resultados}

Habiendo sido establecidos los criterios de operacionalización utilizados, en esta sección se muestra la brecha kilocalórica estimada para el periodo trabajado, seguido por las brechas con los tres grupos de alimentos, dados estos resultados los criterios de evaluación permitirán hacer conclusiones sobre el estado de la SA en el país, su relación con el comercio exterior y los posibles escenarios futuros.

\subsection{Brecha Kilocalórica}

La Tabla 4 muestra los requerimientos energéticos per cápita diarios separados por género y a nivel departamental, para los años 2001 y 2012. El requerimiento promedio de energía para el año 2001 es de 2395 kcal y varía marginalmente para el año 2012 a 2386 kcal, esto puede atribuirse a la migración rural-urbana de los últimos treinta años.

Romero (2012) muestra que en 1976 la población urbana era el 41,3\% de la población nacional, pasando a un 60,6\% en 1996. Según los datos de los censos, ya en el 2001 el $62 \%$ de la población vivía en el área urbano, para el año 2012 se incrementa a 67\%. Podemos notar que Bolivia presenta un proceso de urbanización creciente, debido a que en el área rural el

agrícolas no industriales. Como también: extracción de maderas, desarrollo de plantaciones y otros productos de silvicultura del grupo silvicultura y pesca. Para cada año se descontaron distintos niveles debido a que, como es de esperarse, la participación de estos grupos fluctúa en el tiempo.

34 Se toma la producción interna de alimentos, descontando el consumo intermedio y el destinado a actividades pecuarias.

35 Sub-registro: es una variable proxi al contrabando que utiliza el INE. 
requerimiento calórico es mayor al registrado en el área urbana la migración campo-ciudad, en agregado genera que el requerimiento promedio disminuya.

Tabla 4

Requerimientos Calóricos

\begin{tabular}{lccc|ccc}
\hline & \multicolumn{2}{c}{ K001 } \\
& \multicolumn{2}{c}{ Kcal/día } & \multicolumn{4}{c}{$\begin{array}{c}\text { 2012 } \\
\text { Kcal/día }\end{array}$} \\
\hline \hline Bolivia & Hombres & Mujeres & Promedio & Hombres & Mujeres & Promedio \\
\hline La Paz & 2647 & 2143 & 2395 & 2637 & 2134 & 2386 \\
Oruro & 2648 & 2144 & 2396 & 2648 & 2144 & 2396 \\
Potosí & 2664 & 2156 & 2410 & 2653 & 2148 & 2401 \\
Cochabamba & 2729 & 2210 & 2470 & 2711 & 2195 & 2453 \\
Chuquisaca & 2640 & 2137 & 2389 & 2620 & 2121 & 2371 \\
Tarija & 2690 & 2178 & 2434 & 2673 & 2164 & 2419 \\
Pando & 2632 & 2131 & 2382 & 2627 & 2127 & 2377 \\
Beni & 2649 & 2145 & 2397 & 2649 & 2145 & 2397 \\
Santa Cruz & 2593 & 2100 & 2347 & 2586 & 2090 & 2338 \\
\hline Fuente: Elaboracy & 2576 & 2085 & 2331 & 2563 & 2075 & 2319 \\
\hline
\end{tabular}

Fuente:Elaboración de los autores en base a: los valores es timados con POPER.

La Tabla 4 ilustra la brecha entre el requerimiento calórico calculado y la oferta alimentaria ponderada en kilocalorías (Véase, Anexo 3). Como puede verse el año 1990 la ingesta efectiva de un boliviano promedio era de 1959 (kcal/día) mientras el requerimiento 2386 (kcal/día). Para el año 2000 la ingesta efectiva alcanza 2130 (kcal/día) y el requerimiento se incrementa a una menor tasa hasta llegar a ser 2395 (kcal/día) ya el año 2012 la ingesta llega a 2146 (kcal/día) y el requerimiento a 2386 (kca/día).

Como puede apreciarse en el Gráfico 2, es de esperar que ceteris paribus la ingesta llegue a abastecer al requerimiento, si continúa creciendo como hasta la actualidad. Sin embargo dado los escenarios establecidos, la oferta no abastece el requerimiento $(O<\bar{e})$ por lo que esto coloca al país en una situación de Requerimiento Calórico Insuficiente. Para ver los valores, (Véase el Anexo 4). El siguiente acápite revisará los requerimientos nutricionales, y uniendo estos criterios se establecerá el escenario existente en el país.

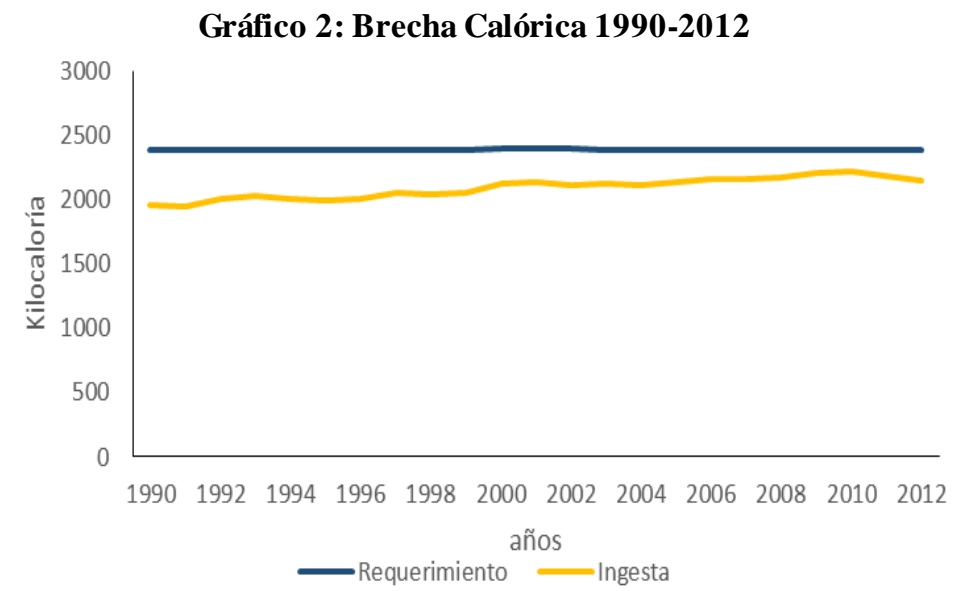

Fuente: Elaboración de los autores en base al requerimiento kilocalórico simulados en comparación a la oferta alimentaria encontrada por medio de los datos extraídos de las Matices Insumo-Producto del INE (1990-2012) y ponderada en kilocalorías, descontando el desperdicio de ésta. 


\subsection{Brechas Nutricionales}

Para este apartado el requerimiento energético fue distribuido según la cantidad de ingesta óptima por tipo de alimento (carbohidratos, proteínas, frutas y verduras) y ponderado en valor para ser comparándolo con la oferta de dichos grupos de alimentos.

Se analiza la oferta alimentaria por grupo de alimento, en la que se muestra la oferta siguiendo la ecuación (10), es decir incluidas las importaciones, pero también se ha proyectado, la producción interna sin las exportaciones, para aportar al análisis la importancia del sector externo dentro de la seguridad alimentaria boliviana.

\subsubsection{Hidratos de Carbono ${ }^{36}$}

Como puede verse en el Gráfico 3 para el grupo de hidratos de carbono las dimensiones de disponibilidad, soberanía alimentaria, estabilidad y consumo y utilización biológica se cumplen. Sin embargo existe dependencia hacia la producción extranjera, debido a las preferencias de los agentes hacia el consumo de estos alimentos, específicamente por cereales y azúcares, ambos dentro del grupo de carbohidratos.

Evaluando la disponibilidad se observa que la oferta (producción e importación) abastece el requerimiento mínimo y el país no tiene déficit en este grupo de alimento. Bajo el supuesto de ceteris paribus la producción se incrementa, pero dado el crecimiento poblacional, a nivel per cápita a nivel per cápita se mantiene constante, la oferta alimentaria tiene una tendencia creciente, a pesar de que el requerimiento sea abastecido con la producción, se recurre a la importación.

Dado esto, se podría concluir que las importaciones no son necesarias para abastecer el requerimiento mínimo, sin embargo al observar que la participación de las importaciones dentro de la oferta ha sido creciente todos estos años. En 1990 las importaciones representaban tan sólo el $19 \%$ de la oferta ya en el año 2000 llegan a $52 \%$ y fluctúan levemente hasta el año 2012 donde representan el 51\%.

Con respecto a la soberanía alimentaria existe autarquía ya que el requerimiento es abastecido con un excedente del $23 \%$ por la producción interna. Lo cual implica a su vez que la producción interna es suficiente. El comercio exterior existente favorece la soberanía alimentaria ya que se realiza por razones de ventajas comparativas y diversifica la oferta interna.

Respecto a la estabilidad de la oferta de carbohidratos en el tiempo, como puede verse que si bien la producción interna tiene algunas fluctuaciones. En los años 2000-2003 la producción cae abruptamente $91 \%$ 95\% y 94\%, respectivamente pero esta disminución se recupera representando el año 2012 el 115\% del requerimiento. Si se incluyen las importaciones la oferta alimentaria es estable en el tiempo.

Dentro de la dimensión de consumo y utilización biológica, la línea alimentaria nutricional entendida como el requerimiento de carbohidratos, es inferior a la oferta ya que la oferta la supera en 42\%, 43\% y 66\% para los años 1990, 2000 y 2012, respectivamente. Dados los datos proyectados, se espera que para el año 2030 la producción que se destina para el consumo interno exceda en $22 \%$ el requerimiento.

\footnotetext{
36 También llamados azúcares, son compuestos que proporcionan energía al cuerpo. Están presentes en alimentos de origen vegetal; por ejemplo: cereales, que incluyen al arroz, trigo y maíz; tubérculos, como la papa y el camote y en algunas frutas, como la manzana, la guayaba y el plátano.
} 


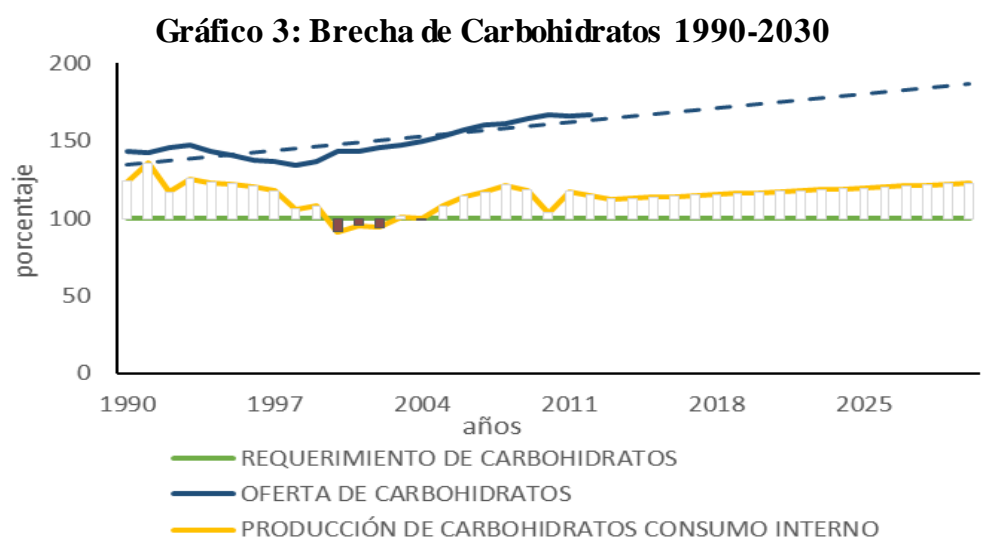

Fuente: Elaboración de los autores en base a la oferta de carbohidratos per cápita encontrada por medio de los datos extraídos de las Matices Insumo-Producto del INE (1990-2012) en comparación al requerimiento dado el nivel calórico simulado dado el requerimiento según el plato de Harvard adaptado para el país, ambos ponderados según la metodología del INE y proyectados en base a las proyecciones del BCB (2013-2030).

\subsubsection{Proteínas ${ }^{37}$}

Como puede verse en el Gráfico 4 dentro del grupo de proteínas, las dimensiones de disponibilidad, soberanía, estabilidad y consumo y utilización biológica no se cumplen. Las proteínas de origen animal y sus derivados han incrementado estos últimos años debido a la producción del Oriente. Por lo que es muy posible que el requerimiento de carnes se abastezca, debido al crecimiento en ganado vacuno lo que conllevará a que la producción de lácteos también se amplíe.

La dimensión de disponibilidad de alimentos no se cumple. Puede verse que la oferta alimentaria de proteínas, es decir el agregado de la producción que se destina al consumo interno y las importaciones, muestra como la brecha disminuye con el tiempo, pero sin llegar a cerrarse para el 2030 asumiendo ceteris paribus.

Al respecto de la soberanía, a pesar de no existir autarquía evaluando el comercio exterior puede apreciarse que en lo que respecta a leguminosas, la variación de la oferta que depende de la producción interna estará en función de la demanda externa y sus fluctuaciones. Las proteínas de origen vegetal son significantes dentro de la producción, pero del total producido, el $80 \%$ se destina principalmente a torta de soya y la mayoría se exporta para alimento balanceado de ganado en el exterior; el 20\% restante se convierte en aceite y de esta cantidad, sólo una quinta parte se queda en el país.

Para evaluar la estabilidad, si bien no existe disponibilidad de alimentos, la producción interna no presenta grandes fluctuaciones, así como también la oferta. La producción interna representa el $49 \%$ para el año 1990 y se incrementa a una tendencia creciente debido a la favorable evolución de la matriz productiva alimenticia por trasladarse al oriente. El año 2000 ésta representa el 53\% y 65\% el 2012. Las importaciones no tienen una participación muy alta en 1990 representaban el 3\% del requerimiento, ya en el año 2000 se incrementan hasta un $8 \%$ y el año 2012 un $7 \%$.

Respecto al consumo y utilización biológica nutricionalmente para los años 1990, 2000 y 2012 el déficit es de 48\%, 39\% y 28\% respectivamente. La producción interna responde a

37 Son sustancias que permiten el crecimiento del organismo. La mayor parte de éstas se encuentran en alimentos de origen animal y en plantas leguminosas. 
presiones internacionales y el sector industrial obtiene mayores utilidades en el mercado externo por lo que se prioriza la exportación.

Gráfico 4: Brecha de Proteínas 1990-2030

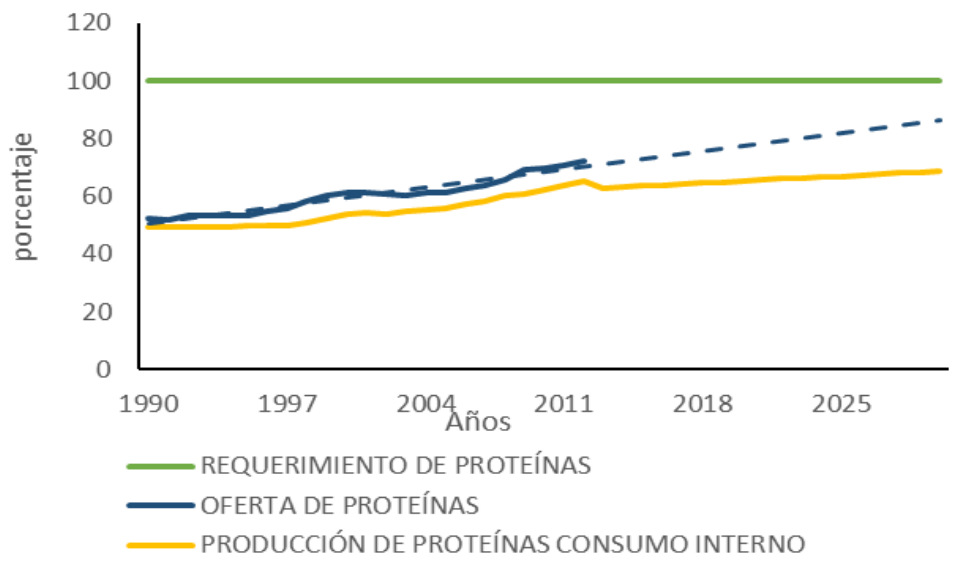

Fuente: Elaboración de los autores en base a la oferta de proteínas per cápita encontrada por medio de los datos extraídos de las Matices Insumo-Producto del INE (1990-2012) en comparación al requerimiento dado el nivel calórico simulados dado el requerimiento según el plato de Harvard adaptado para el país, ambos ponderados según la metodología del INE y proyectados en base a las proyecciones del BCB (2013-2030).

\subsubsection{Frutas y verduras}

Como puede verse en el Gráfico 5, no se cumplen las dimensiones de disponibilidad, soberanía, estabilidad y consumo y utilización biológica. Sin embargo el boliviano promedio no tiene la costumbre de comer frutas y verduras, por lo que un incremento de la oferta no solucionaría, por sí solo, el problema nutricional que existe en el país.Las importaciones representan casi el $50 \%$ del total de la oferta interna por lo que el país depende de la producción internacional para abastecerse, a pesar de cumplir con las condiciones climáticas para la producción de este tipo de alimentos.

Respecto a la disponibilidad la oferta alimentaria (importaciones y la producción destinada al consumo interno) abastece tan sólo el $13 \%$ del requerimiento mínimo de frutas y verduras, incrementándose en los años 2000-2003 hasta un 23\%, debido a una mejora en la productividad de este tipo de cultivos, sin embargo debido a que la población no tiene la costumbre de ingerir estos alimentos el incremento en la productividad se ve reflejado en el aumento de la exportación de este grupo de alimento.

No existe soberanía alimentaria ya que al margen de no cumplirse la autarquía para el grupo de frutas y verduras de alimentos existe un déficit. La producción interna representa alrededor del 9\% del requerimiento, proyectando la producción bajo las condiciones actuales para el año 2030 la producción cubrirá sólo un 10\% del requerimiento. En 1990 las importaciones representan el 5\% del requerimiento, aumentando hasta el año 2000 para mantenerse constantes hasta el 2003 representando 12\% del requerimiento.

Tanto para la dimensión de estabilidad como para la de consumo y utilización biológica, es necesario que la dimensión de disponibilidad se cumpla. Al no ser así, se puede concluir que si bien la producción no fluctúa en el tiempo, manteniéndose constante alrededor del $10 \%$ del requerimiento nutricional mínimo, ésta deberá incrementarse. Por su parte a nivel nutricional el requerimiento de frutas y verduras sobrepasa la oferta alimentaria con $86 \%, 76 \%$ y $82 \%$ para los años 1990, 2000 y 2012 respectivamente. 


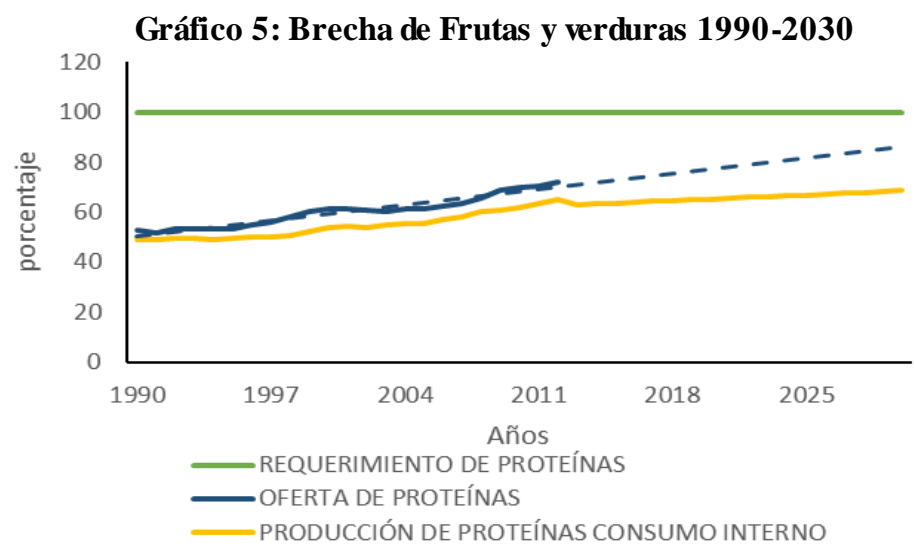

Fuente: Elaboración de los autores en base a la oferta de frutas y verduras per cápita encontrada por medio de los datos extraídos de las Matices Insumo-Producto del INE (1990-2012) en comparación al requerimiento dado el nivel calórico simulado el requerimiento según el plato de Harvard adaptado para el país, ambos ponderados según la metodología del INE y proyectados en base a las proyecciones del BCB (2013-2030).

\section{Conclusiones}

A nivel calórico la oferta interna no abastece el requerimiento energético. Los escenarios encontrados para el país muestran que no se genere autarquía en el país, provocando una importante, aunque decreciente, dependencia externa del país. Por grupo de alimento, sólo el grupo de carbohidratos cumple con las dimensiones estudiadas. Tanto el grupo de proteínas, como el de frutas y verduras, no cumplen con ninguna de las dimensiones.

Para el grupo de carbohidratos la oferta alimentaria interna, sin necesidad de importaciones cumple con el requerimiento nutricional mínimo. Sin embargo es de notar la dependencia que se tiene hacia el sector extranjero, es debida a las preferencias de los bolivianos por el consumo de este grupo de alimento.

El grupo de proteínas prioriza las exportaciones, debido a que los precios internacionales generan mayores utilidades al sector industrial, desabasteciendo el mercado interno y generando déficit dentro del requerimiento nutricional.

Tradicionalmente los bolivianos no están acostumbrados al consumo de frutas y verduras. Las importaciones de éstas representan alrededor del 50\% de la oferta interna. Este grupo está caracterizado por una producción de alimentos insuficiente y compensada por la importación, aun así no llega a abastecer el mínimo. 


\section{Bibliografía}

[1] Barret, C. (2002). "Food security and food assistance programs", Capítulo 40 del Handbook of Agricultural Economics, Volumen 2.

[2] De la Vega, A.. (2004). Seguridad Alimentaria. diciembre 2, 2004, de Gestiopolis Sitio web: http://www.gestiopolis.com/canales3/ger/segalim.htm

[3] Deaton, A. (1997). Understanding consumption, Oxford. Clarendon Press.

[4] Elmadfa., Leitzmann., y Ernaehrung des Menschen (1990) ¿Qué componentes se distinguen en los alimentos?

[5] FAO/OMS/UNU (1985). Necesidades de energía y proteína. Informe de una reunión consultiva conjunta FAO-OMS-UNU de expertos. Organización Mundial de la Salud. Serie de informes técnicos. OMS, Ginebra.

[6] FAO/WHO/UNU (2001). Human Energy Requirements.

[7] FAO (2001). The State of Food Insecurity in the World.

[8] FAO (2001). Perfiles nutricionales por países: Bolivia.

[9] FAO/PMA(2002). Análisis y Cartografía de la Vulnerabilidad a la Inseguridad Alimentaria en Bolivia.

[10] FAO/OMS (2004). Principios y aplicación de las nuevas necesidades de energía según el Comité de Expertos.

[11] FAO (2006). Seguridad Alimentaria: Informe de Políticas.

[12] FAO (2008). Metodología de la FAO para medir la privación de alimentos: Actualizando las necesidades energéticas mínimas.

[13] FAO (2013). El estado de la inseguridad alimentaria en el mundo: Las múltiples dimensiones de la seguridad alimentaria.

[14] Gros, R. y Schoeneberger, H. (2002).Las cuatro dimensiones de la seguridad alimentaria y nutricional: Definiciones y Conceptos. GTZ.

[15] Haquim, D. (2009). Seguridad Alimentaria. pp. 244-260.

[16] Haddinott, J. (1999) Operationalizing household food security in development projects: an introduction. In: technical guide for food security. Whasington.

[17] Izquierdo, S. (2014). Evaluación del crecimiento de niños y adolescentes en Bolivia (no publicado).

[18] Instituto Nacional de Estadística (s/f). Metadato de Cuentas Nacionales. Metodología Insumo Producto.

[19] Ismail, N., Chee, S., Roslee, R. y Zawiah, H. (1998). Predictive equations for the estimation of basal metabolic rate in Malaysian adults. Malasia.

[20] Mallea, I. (2010). Situación actual y prioridades básicas de la seguridad alimentaria nutricional en Bolivia. Mayo, 27, 2010.

[21] Ormachea, E. (2010). Soberanía y Seguridad Alimentaria en Bolivia: Políticas y Estado de la situación. ANÁLISIS. 4 (4), 11-13.

[22] Romero, C. (2012). Crisis, Seguridad y Soberanía Alimentaria en América Latina y Bolivia: De las causas y efectos a las políticas públicas. La Paz: KIPUS.

[23] Rosegrant, M.W., Ringler, C., Msangi, S., Cline, SA y Sulser, TB (2005). International Model for Policy Analysis of Agricultural Commodities and Trade (IMPAC-WATER): Model Description.

[24] Salcedo, S. (2005). Políticas de la Seguridad Alimentaria en los países de la Comunidad Andina. Marco teórico de la Seguridad Alimentaria.

[25] Seckler, D., Amarasinghe, U., Molden, D., de Silva, R., Barker, R., (1998). World water demand and supply, 1990 to 2025: scenarios and issues. Research Report $N^{\circ} 19$, International

[26] Shapouri, S. y Rosen, S. (2005). Food Security Assessent. Electronic Outlook Report fron the Economic Research Service. GFA-16. 
[27] Schofield, W. Schofield, E y James, W. (1985). Predicting basal metabolic rate: new standards and review of previous work.

[28] UDAPE (2007). Estado del arte de la seguridad alimentaria nutricional boliviana.

[29] UNICEF (1990). The State of the world's children 1990.

[30] Water Management Institute (IWMI), Colombo, Sri Lanka. FAO. (1993). The World Food Model-Model Specifications ESC/M/93/1. 


\section{Anexo 1: Tipos de índices para cuantificar la seguridad alimentaria}

Salcedo (2005) presenta algunas de las aproximaciones propuestas en la literatura para medirlo, considerando tanto medidas sobre la insuficiencia de ingreso y aspectos nutricionales, como medidas antropométricas. El indicador más usado para medir el estado de la IA en el mundo es la prevalencia de la subnutrición (FAO 2003) (Salcedo, 2005).

- Indicadores relacionados con la salud:

- Porcentaje de niños con bajo peso al nacer (menos de 2500 g);

- Porcentaje de niños menores de 5 años con desnutrición;

- Porcentaje de escolares de 7 años con déficit de talla para la edad en relación con los valores de referencia;

- Tasas de mortalidad infantil y pre-escolar;

- Porcentaje de adultos con déficit energético crónico, según el Índice de Masa Corporal (IMC).

- Indicadores e instrumentos recomendados por la FAO (2013):

- Instrumento de "diversidad alimentaria ${ }^{38 " . ~ E s t e ~ i n s t r u m e n t o ~ u s a ~ u n ~ m e ́ t o d o ~ d e ~}$ recordatorio cualitativo y abierto para recopilar información sobre todos los alimentos consumidos a lo largo de las 24 horas precedentes a la encuesta ${ }^{39}$, los cuales se clasifican posteriormente en grupos de alimentos normalizados;

- La nueva Escala del Hambre en los Hogares (HHS) es un indicador para evaluar el hambre en zonas con gran $\mathrm{IA}^{40}$;

- La Escala del Componente de Acceso de la IA en el hogar (HFIAS) (inglés) compuesta por nueve preguntas - sirvió de base para la HHS. La HFIAS no ha sido validada para su uso transcultural;

La Escala Latinoamericana y Caribeña de SA (ELCSA) un instrumento de medición normalizado y validado de uso generalizado en América Latina y el Caribe, se está adaptando en la actualidad para uso en otras partes del mundo.

38 La diversidad alimentaria, se define como el número de grupos de alimentos consumidos durante un periodo determinado.

39 El cuestionario puede realizarse tanto a nivel individual como a nivel de los hogares. En la guía se describe cómo adaptar la herramienta a los sistemas alimentarios locales. En los análisis se incluye el cálculo de los valores de diversidad alimentaria, de los porcentajes de hogares o individuos que consumen ciertos grupos de alimentos con interés nutricional (por ejemplo; la vitamina A) y de los patrones de diversidad alimentaria. La información generada a partir del instrumento de la diversidad alimentaria es particularmente útil para desarrollar políticas agrícolas y programas de nutrición eficaces para proporcionar y promover alimentos nutritivos con el fin de garantizar la seguridad alimentaria y nutricional. Además, el instrumento puede usarse con rapidez y facilidad, y puede integrarse fácilmente en los protocolos de evaluación del impacto.

40 Elaborada por el proyecto de Asistencia Técnica sobre Alimentos y Nutrición (FANTA) en colaboración con la FAO. 


\section{Anexo 2: Distribución del Nivel de Actividad Física Según Área Geográfica}

TABLA: Residencia urbana con actividad ligera

\begin{tabular}{|l|r|r|r|r|}
\hline ACTIVIDAD & $\begin{array}{r}\text { Tiempo } \\
\text { (horas) }\end{array}$ & $\begin{array}{r}\text { Costo de } \\
\text { energía }\end{array}$ & $\begin{array}{r}\text { Tiempox } \\
\text { Costo }\end{array}$ & $\begin{array}{r}\text { NiveI de } \\
\text { actividad } \\
\text { física }\end{array}$ \\
\hline Dormir & 7 & 1 & 7 & \\
Comer & 1 & 1,5 & 1,5 & \\
Cuidado personal (vestirse, bañarse) & 1 & 1,5 & 1,5 & \\
Movilizarse en autobús & 2 & 1 & 2 & \\
Caminar y/o levantar objetos ligeros & 2,5 & 3 & 7,5 & \\
Sentarse & 1,5 & 1,5 & 9 & \\
Realizar tareas domésticas & 0,5 & 3,0 & 4,5 & \\
Ejercicio aeróbico de baja intensidad & 2,5 & 1,0 & 1,5 & \\
Actividades de ocio (ver la televisión, leer) & $\mathbf{2 4}$ & $\mathbf{1 9 , 9}$ & $\mathbf{3 7 , 5}$ & $\mathbf{1 , 5 6}$ \\
TOTAL & & & \\
\hline
\end{tabular}

Fuente: Elaboración de los autores en base a FAO/OMS(2004)

TABLA: Residencia urbana con actividad más que ligera

\begin{tabular}{|c|c|c|c|c|}
\hline ACTIVIDAD & $\begin{array}{r}\text { Tiempo } \\
\text { (horas) }\end{array}$ & $\begin{array}{r}\text { Costo de } \\
\text { energía }\end{array}$ & $\begin{array}{r}\text { Tiempo } x \\
\text { Costo }\end{array}$ & $\begin{array}{r}\text { Nivel de } \\
\text { actividad } \\
\text { física }\end{array}$ \\
\hline Dormir & 7 & 1 & 7 & \\
\hline Comer & 1 & 1,5 & 1,5 & \\
\hline Cuidado personal (vestirse, bañarse) & 1 & 2 & 2 & \\
\hline Movilizarse en autobús & 7 & 2,8 & 19,6 & \\
\hline Caminar y/o transportar objetos ligeros & 2,5 & 2,5 & 6,3 & \\
\hline Sentarse & 3 & 1,6 & 4,8 & \\
\hline Realizar tareas domésticas & 2,5 & 1,2 & 3 & \\
\hline TOTAL & 24 & 12,6 & 44,2 & 1,84 \\
\hline
\end{tabular}

Fuente: Elaboración de los autores en base a FAO/OMS(2004)

TABLA: Residencia rural con actividad ligera

\begin{tabular}{|c|c|c|c|c|}
\hline ACTIVIDAD & $\begin{array}{r}\text { Tiempo } \\
\text { (horas) }\end{array}$ & $\begin{array}{c}\text { Costo de } \\
\text { energía }\end{array}$ & $\begin{array}{r}\text { Tiempo } x \\
\text { Costo }\end{array}$ & $\begin{array}{l}\text { Nivel de } \\
\text { actividad } \\
\text { física }\end{array}$ \\
\hline Dormir & 7 & 1 & 7 & \\
\hline Comer & 1 & 1,5 & 1,5 & \\
\hline Cuidado personal (vestirse, bañarse) & 1 & 2 & 2 & \\
\hline Movilizarse en autobús & 8 & 2,5 & 20 & \\
\hline Caminar y/o transportar objetos ligeros & 0,5 & 5 & 2,5 & \\
\hline Sentarse & 1,5 & 1,5 & 2,25 & \\
\hline Realizar tareas domésticas & 3 & 2,2 & 6,6 & \\
\hline Actividades de ocio (ver la televisión, leer) & 2 & 1,2 & 2,4 & \\
\hline TOTAL & 24 & 16,9 & 44,3 & $\mathbf{1 , 8}$ \\
\hline
\end{tabular}

Fuente: Elaboración de los autores en base a FAO/OMS(2004)

TABLA: Residencia urbana con actividad más que ligera

\begin{tabular}{|c|c|c|c|c|}
\hline ACTIVIDAD & $\begin{array}{r}\text { Tiempo } \\
\text { (horas) }\end{array}$ & $\begin{array}{l}\text { Costo de } \\
\text { energía }\end{array}$ & $\begin{array}{r}\text { Tiempo } x \\
\text { Costo }\end{array}$ & $\begin{array}{r}\text { Nivel de } \\
\text { actividad } \\
\text { física } \\
\end{array}$ \\
\hline Dormir & 7 & 1 & 7 & \\
\hline Comer & 1 & 2 & 2 & \\
\hline Cuidado personal (vestirse, bañarse) & 1 & 1,5 & 1,5 & \\
\hline Movilizarse en autobús & 2 & 2,1 & 4,2 & \\
\hline Caminar y/o levantar objetos ligeros & 2 & 4,2 & 8,4 & \\
\hline Sentarse & 2 & 3,6 & 7,2 & \\
\hline Realizar tareas domésticas & 3,5 & 2,3 & 8,1 & \\
\hline Ejercicio aeróbico de baja intensidad & 3,5 & 1,6 & 5,6 & \\
\hline Actividades de ocio (ver la televisión, leer) & 2 & 1,6 & 3,2 & \\
\hline TOTAL & 24 & 19,9 & 47,2 & 1,96 \\
\hline
\end{tabular}

Fuente: Elaboración de los autores en base a FAO/OMS(2004) 


\section{Anexo 3: Conversión de la Oferta de Valor a kilocalorías}

Para comparar el requerimiento energético per cápita, con la oferta medida en valor, se realizan las siguientes conversiones:

Debido a que, para contabilizar la oferta se usaron las matrices insumo producto del INE, se tomó la metodología del para que estos datos tengan la misma unidad de medida; por lo que dada la ecuación (1):

$\frac{v}{p}=\operatorname{tn}$

Como la serie es anual para tener la ingesta calórica diaria, se tiene:

$\frac{t n}{365}=t n d i ́ a$

Dado que 1tn=1000000gr, la conversión genera:

$g r=\operatorname{tn}$ día $* 1000000$

Por último, dado:

$E^{\prime} \mathrm{Kcal}=$ Kilo caloria del producto cada $100 \mathrm{gr}$

Se Tiene:

$k c a l=g r$ día* $E^{\prime} / 100 \mathrm{gr}$

TABLA: oferta anual en kcal

\begin{tabular}{l|lllllllllll} 
año & 1990 & 1991 & 1992 & 1993 & 1994 & 1995 & 1996 & 1997 & 1998 & 1999 & 2000 \\
\hline kcal & 1959 & 1953 & 2006 & 2036 & 2012 & 2001 & 2014 & 2050 & 2042 & 2060 & 2130
\end{tabular}

\begin{tabular}{l|llllllllllll} 
año & 2001 & 2002 & 2003 & 2004 & 2005 & 2006 & 2007 & 2008 & 2009 & 2010 & 2011 & 2012 \\
\hline kcal & 2136 & 2111 & 2122 & 2115 & 2138 & 2163 & 2165 & 2172 & 2215 & 2227 & 2187 & 2146
\end{tabular}

Fuente:Elaboración de loa autores. 
Anexo 4: Valores de la ingesta y el requerimiento energético

TABLA: Brecha Calórica 1990-2012

\begin{tabular}{l|lllllllllll} 
Año & 1990 & 1991 & 1992 & 1993 & 1994 & 1995 & 1996 & 1997 & 1998 & 1999 & 2000 \\
\hline Requerimiento & 2386 & 2387 & 2388 & 2389 & 2390 & 2391 & 2392 & 2392 & 2393 & 2394 & 2395 \\
\hline Ingesta & 1959 & 1953 & 2006 & 2036 & 2012 & 2001 & 2014 & 2050 & 2042 & 2060 & 2130
\end{tabular}

\begin{tabular}{l|llllllllllll} 
Año & 2001 & 2002 & 2003 & 2004 & 2005 & 2006 & 2007 & 2008 & 2009 & 2010 & 2011 & 2012 \\
\hline Requerimiento & 2396 & 2395 & 2394 & 2393 & 2392 & 2392 & 2391 & 2390 & 2389 & 2388 & 2387 & 2387 \\
\hline Ingesta & 2136 & 2111 & 2122 & 2115 & 2138 & 2163 & 2165 & 2172 & 2215 & 2227 & 2187 & 2146
\end{tabular}

Fuente: Elaboración de los autores en base al requerimiento kilocalórico simulados en comparación a la oferta alimentaria encontrada por medio de los datos extraídos de las Matices Insumo-Producto del INE (1990-2012) y ponderada en kilocalorías, descontando el desperdicio de ésta. 